\title{
The concept of contradiction in ergonomics practice
}

\author{
Dimitris Nathanael $^{\mathrm{a},}{ }^{*}$, Nikos Zarboutis $^{\mathrm{b}}$ and Nicolas Marmaras ${ }^{\mathrm{a}}$ \\ ${ }^{a}$ Ergonomics Unit, School of Mechanical Engineering, National Technical University of Athens, Iroon \\ Plotechneiou 9, GR-157 80, Zografos, Greece \\ ${ }^{\mathrm{b}}$ WORKSYSTEMS Engineering, Mavromichali 167, GR-114 72, Athens, Greece
}

\begin{abstract}
The present communication deals with the methodology of the ergonomics field intervention process. It proposes an operationalized version of work analysis in terms of contradictions. The aim is to demonstrate that such a dialectic tool and method of representation may assist the ergonomist to frame the essence of a work activity in practical terms, swiftly and in a manner that preserves its multifaceted unity. The proposed method is inspired by two theoretical constructs (i) contradiction as used in Cultural Historical Activity Theory and (ii) regulation, as developed and used by the francophone tradition of ergonomics of activity. Two brief examples of its use are presented and a discussion is made on further developments and possible pitfalls.
\end{abstract}

Keywords: intervention, contradiction, conflict, activity analysis, regulation

\section{Introduction}

Intervening in a real work-situation is almost always a challenging experience. Where does one start from? In most cases there is of course an explicit request, an initial problem to address, but still, how does one go about tackling a complex reality? In most real work situations, concerns, complaints, dilemmas and stakes are so intermingled (sometimes positive in one respect and negative in another) that when one tries to get hold of them, he rapidly feels overwhelmed by the complexity.

The traditional way to proceed is to try to thematise (i.e. analyze according to particular topics). One will often start by decomposing elements of a work situation (either in structural, functional or temporal terms). For example, a clerical work will typically be decomposed in the physical morphology of the workstation, the informational demands of the work and probably the organizational imperatives. The three components will be examined one by one, the first dealing with the aspects of physical health, the second with aspects of mental fatigue and/or mistakes, while the third possibly with psychosocial stress etc.
This strategy, although systematic, tends to lead to a fragmented view of work. This fragmentation often results to incremental improvements in each of the decomposed elements. This is in line with ergonomics \& human factors handbooks but often fail to identify key issues or even the essence of a particular work situation. For example, in the clerical task, the complex interaction between cognitive effort, informational demands, and postural comfort may often be missed through this approach. This is one of the reasons why the so called "ergonomic standards or guidelines" are marginally useful in practice.

In the same manner, when a work situation is decomposed in temporal rather than thematic terms e.g. in phases, one may fail to identify important interdependencies or conflicts between determinants that manifest themselves at different time frames (e.g. long term health vs short term comfort or production quantity vs. safety etc). For example observed inefficiencies in a route taken over and over again may hide a safety concern (warranted or not).

The above critique is not new. In fact it is part of a continuous debate concerning the systemic epistemology of ergonomics as a discipline. In any case it

*Corresponding author. E-mail: dnathan@central.ntua.gr. 
does not imply that an experienced ergonomist will unavoidably miss such issues once he adopts a decomposition strategy; it merely suggests that decomposition methods do not assist the identification of multi-level or multi-thematic concerns.

The present communication aims to contribute to the methodological toolset of the ergonomics field intervention process by proposing and demonstrating a "hands on" version of the concept of contradiction. We hope to show that a "hands on" dialectic tool and method of representation may help frame the essence of a work activity in practical terms, and in a manner that preserves its multifaceted unity. The proposed conceptual tool is inspired by two theoretical constructs i) contradiction as used in Cultural Historical Activity Theory (CHAT) and ii) regulation, as developed and used by the francophone tradition of ergonomics of activity.

The structure of the communication is as follows: in the following section we briefly present the concepts of contradiction and activity regulation as they have been developed in the context of work analysis and we discuss our hands-on interpretation. A set of case studies will be used afterwards in order to demonstrate our approach to "contradiction analysis" in industrial projects. Finally, we will discuss the possible merits of the approach as well as its pitfalls and the way forward.

\section{The concepts of contradiction and regulation}

There is plethora of notions and concepts in work analysis literature that tackle such phenomena. Apart from contradictions within CHAT (Engeström 1987) and activity analysis in terms of regulation (Faverge et al. 1966) we encounter notions such as balance between internal needs and external demands (Leplat 2000), margins of maneuver (De la Garza \& WeillFassina, 2000), double rationality (Dejours 1995) invariance structure, constraints, boundaries of acceptable performance (Rasmussen et al. 1994), shifting between operating modes (Sperandio, 1972) etc. All these notions, stemming from different traditions of the ergonomics of activity, point to the fact that in concrete situations i) there is never absolute prescription of what needs to be done at any moment and ii) that the working people need to resolve / or are free to exploit the remaining ambiguity. That is, at any moment the workers either take conscious decisions or unconsciously regulate between differing goals and needs.
Contradiction in dialectics is defined as an opposition inherently existing within one realm, one unified force or object. A contradiction is identified whenever two tendencies or forces are interdependent (Unity) yet mutually negate one another. In CHAT contradictions are inspired from dialectic materialism and are defined as objective tensions and conflicts that may both hinder and facilitate the historical development of an activity system (Engeström 1987, Nardi 1996). Typically in CHAT contradictions are identified between elements of the basic structure of the Activity System (i.e. between the subject, object, tools, division of labour, rules and community) or between different activities or within an activity's different developmental stages (Engeström 1987). The theoretical position that contradictions unavoidably exist in work systems and that their acknowledgement may transform an activity system is a major contribution of CHAT in ergonomics thinking.

From a different standpoint, Faverge et al (1966), inspired from cybernetics, proposed the analysis of activity in terms of regulation as one of the three types of activity analysis, the other two being analysis (i) in terms of gestures and (ii) in terms of information. According to Faverge et al. (ibid) workers and/or teams often regulate between partly conflicting or contradictory requirements (e.g. immediate production vs. overall system objectives, or, following of one rule vs. the following of another, or even fatigue vs. safety). The analysis in terms of regulation considers the worker as an active agent who constantly balances between internal resources and external exigencies.

In a way, the concept of regulation in the analysis of activity is a cybernetic reformulation of the basic ergonomics contradiction between task demands and worker abilities. In the classic (i.e. modernist) view, the contradiction "task demands vs. worker abilities" is formulated as a fitting question i.e. "are the worker's abilities enough to deal with the demands of the task? Or, vice-versa, "is the task at hand adapted to the workers abilities?" The classic view is static and unidimensional. The worker - task compatibility model may in fact only find application in simple situations (e.g. when someone needs to lift a heavy object, run a particular distance or perform a set of calculations, etc.).

In the regulation concept the basic opposition (see above) still holds in the form of internal vs. external but regulation adds three important elements: (i) that internal resources are not unidimensional (i.e. they form a system), (ii) that there is always ability to give slack between the two (i.e. temporarily exceed nomi- 
nal limits) and (ii) the worker will freely exploit this slack.

The above concept of regulation can approximate a number of real work situations that are heavily characterized by the principal opposition between individual needs vs external demands. What is more important is that it acknowledges this regulation as a true concern (i.e. an object of work) for the worker. The acknowledgement of this process as an objective and legitimate object of workers' concern was a major contribution of the Francophone School of ergonomics.

In fact, beyond the theoretical subtleties surrounding the above notions, contradictions in activity according to CHAT and activity regulation according to the Francophone school can be understood as the structural and functional equivalents of the same phenomenon. For the sake of clarity, in the remaining of the present communication we will use the term contradiction to refer to the structural view and the overall approach. The term regulation will be used only as the process of balancing between conflicting forces in particular cases.

Two characteristic and well-known types of contradiction in work concern (i) the conflict between personal needs and task demands (Leplat 2000) and (ii) the "trade-off" between efficiency and thoroughness (Hollnagel 2004). The two above bi-poles of opposing forces can be identified in most work situations, and may form the basis of a dialectical approach to work analysis. However such general types of contradiction cannot account for the complexity and plurality of conflicting forces in any concrete work situation.

In a concrete situation contradictions may be identified as a struggle between antagonistic internal resources or between mutually incompatible external exigencies. For example one may identify an intrasubjective contradiction between musculoskeletal health and physiological fatigue as in a typical lifting task. Or one may identify a conflict between personal values that manifest themselves whenever long term health effects are in contrast to financial gains (as in self employed workers). One may thus encounter situations where an internal need is aligned with some external demand but in contradiction to other usually more long term- internal needs. One thus starts to question the concrete usefulness of the personal needs vs. task demands or any other generic type of contradiction. The generic types of contradiction have an undeniable universality but their unreflective use may obscure the particular tensions of any concrete situation.
The core idea in the concept is that human activity can "coarsely but swiftly" be represented by sets of contradictions between the human(s) and his / hers (their) situation. When first encountering an activity system, an analyst may start to recognize such sets of contradictions based on manifestations of instability or irrationality in the system. For example oscillations in performance, differing working styles among workers or teams, disputes between co-workers, inconsistencies in people's accounts etc. all are points of entry for a contradiction analysis. Also, analyzing goal structure vs. organizational structure may provide very fast hints. There is actually no determining sign of an underlying contradiction other than a phenomenal fuzziness or some kind of invariable concern. However, behind such fuzziness or concern there is almost always a choice to be made, a choice that is neither simple nor settled. Moreover, such choices may appear at different levels of a worksystem from intra-individual bodily choices, individual cognitive, motivational, workstation, work-team or organizational level.

In the following section we present two cases where contradiction analysis facilitated problem description and problem solving in our interventions. The descriptions are short and lacking in detail but we hope that the ergonomics practitioner will complement them with parts of his own experience. The case studies come from two different domains, namely:

(a) Silo Trucks drivers 's activity

(b) Wind Turbines maintenance activities

\section{Demonstration of contradiction analysis through case studies}

\subsection{Silo Trucks drivers 's activity}

In a Cement Production factory, cement in powder form is being loaded in silo trucks and transported nationwide to customers' facilities. Twenty-six (26) privately owned Silo Trucks, use a total number of six (6) designated parking spots and load from two (2) loading hoses on a first-come-first-served fashion. Very often, the workplace transport system becomes congested because of trucks moving and stopping within the plant. A request was initiated from the factory management to re-design the vehicle circulation model inside the plant. 
The plant's management has a robust goal structure, mediated by the regulation of multi-leveled contradictions:

(a) At the management level, the production manager regulates truck availability (i.e. orders shipment satisfaction) against workplace transport capacity. This determines the total number of trucks to employ at a given period.

(b) Silo Drivers wish to maximize their own revenue (i.e. deliver as many loads as possible). They regulate the contradiction between adopting the best place in the queue (i.e. minimize waiting time) vis-àvis satisfy personal needs (i.e. work 8 hours per day, spending most of it in driving which values the most, rather than waiting for long which is unproductive, not paid and disturbs their lives and chain of values).

This results to a situation where silo drivers often park their trucks randomly, in order to ensure the best possible position in the queue, balancing between workplace transport policy (i.e. park in nondesignated spots), disturb their work schedule (work plus waiting exceeds $8 \mathrm{~h}$ per day) and suboptimal work experience (too much waiting time; "I want to be on the road"). Amelioration of the workplace transport system passes through an intervention on the regulation of the above mentioned contradictions.

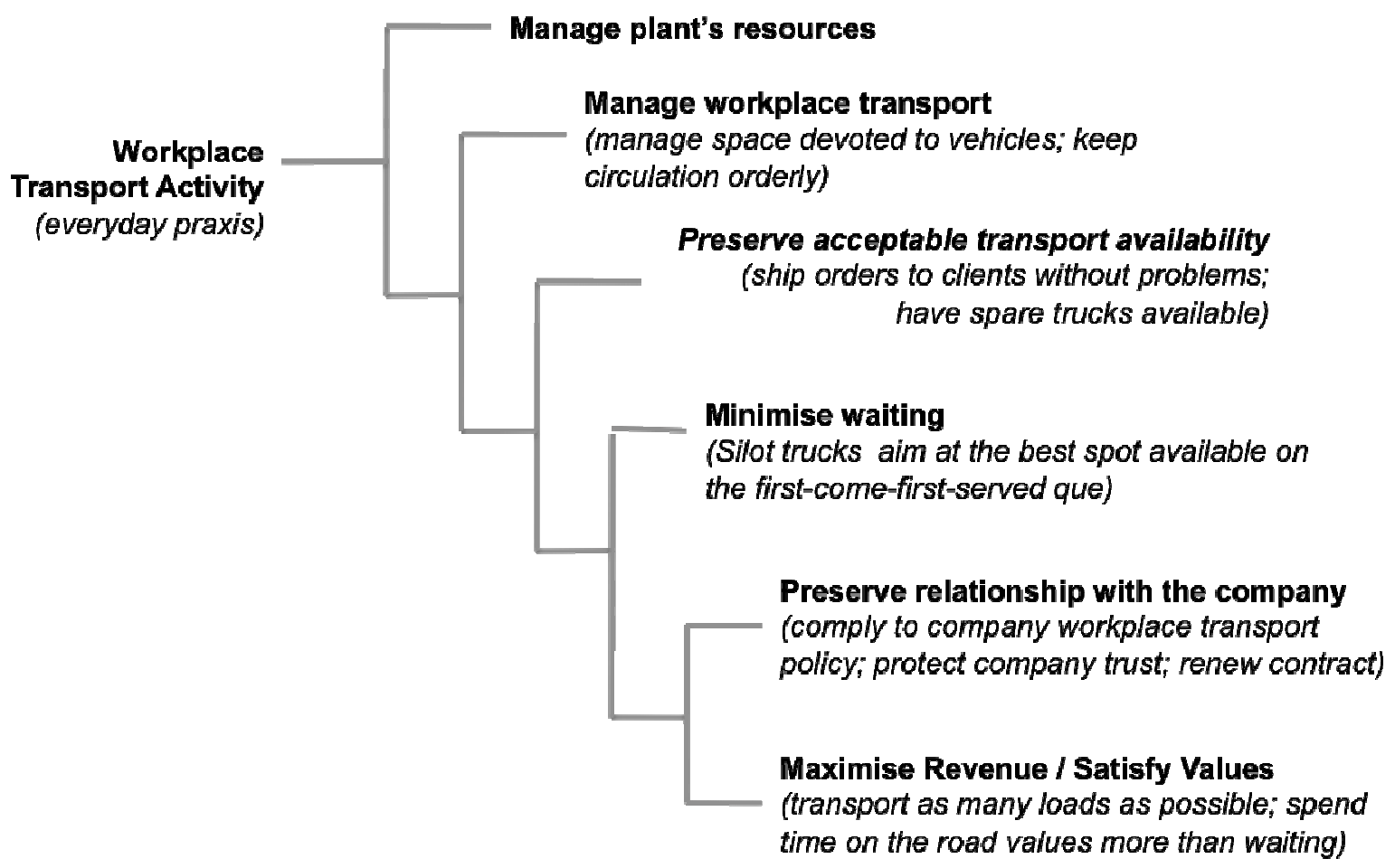

Fig.1. Demonstration of Contradiction Analysis for the Silo Truck Drivers' activities

\subsection{Wind turbine maintenance activity}

Wind turbine maintenance mainly involves the activity of the service dispatcher and the service technicians. The former's activity develops by the regulation of conflicting tasks, set out by interacting:

(a) Upwards, interacting with the Wind Farm owner, regulating the contradiction between profit loss minimization, as service proceeds with the turbine switched off (i.e. arranging that each wind turbines' service will be performed when there is no significant amount of wind resulting to low energy yield), and the need to have the turbine serviced on time. The resolution of this contradiction dynamically defines a time-window where all service activities have to be carried-out, within it.

(b) Downwards, with the service process itself, on an escalating manner:

a. Initially trying to maximize personnel use (i.e. roughly minimize time spent on each $\mathrm{W} / \mathrm{T}$ ), simultaneously ensuring an acceptable quality of service since $\mathrm{s} /$ he has to preserve an availability rate of $99.7 \%$ uptime (availability below $99.7 \%$ results to 
claims, thus interfering with the above mentioned regulation process).

b. Interacting with the technician(s) themselves and the way they perform, value and carry out the service activities.

(c) Each service technician regulates his own contradictions, trying to satisfy his own values (i.e. the sentiment of pride when "his" wind turbines function properly; when the turbine is "clean as new", receiving the appraisal of the customer or peers), competing for more time on every wind turbine, thus asking for thoroughness, against the situation his is into, set dynamically by processes (a) \& (b), resulting to a demand for servicing each turbine as soon as possible.

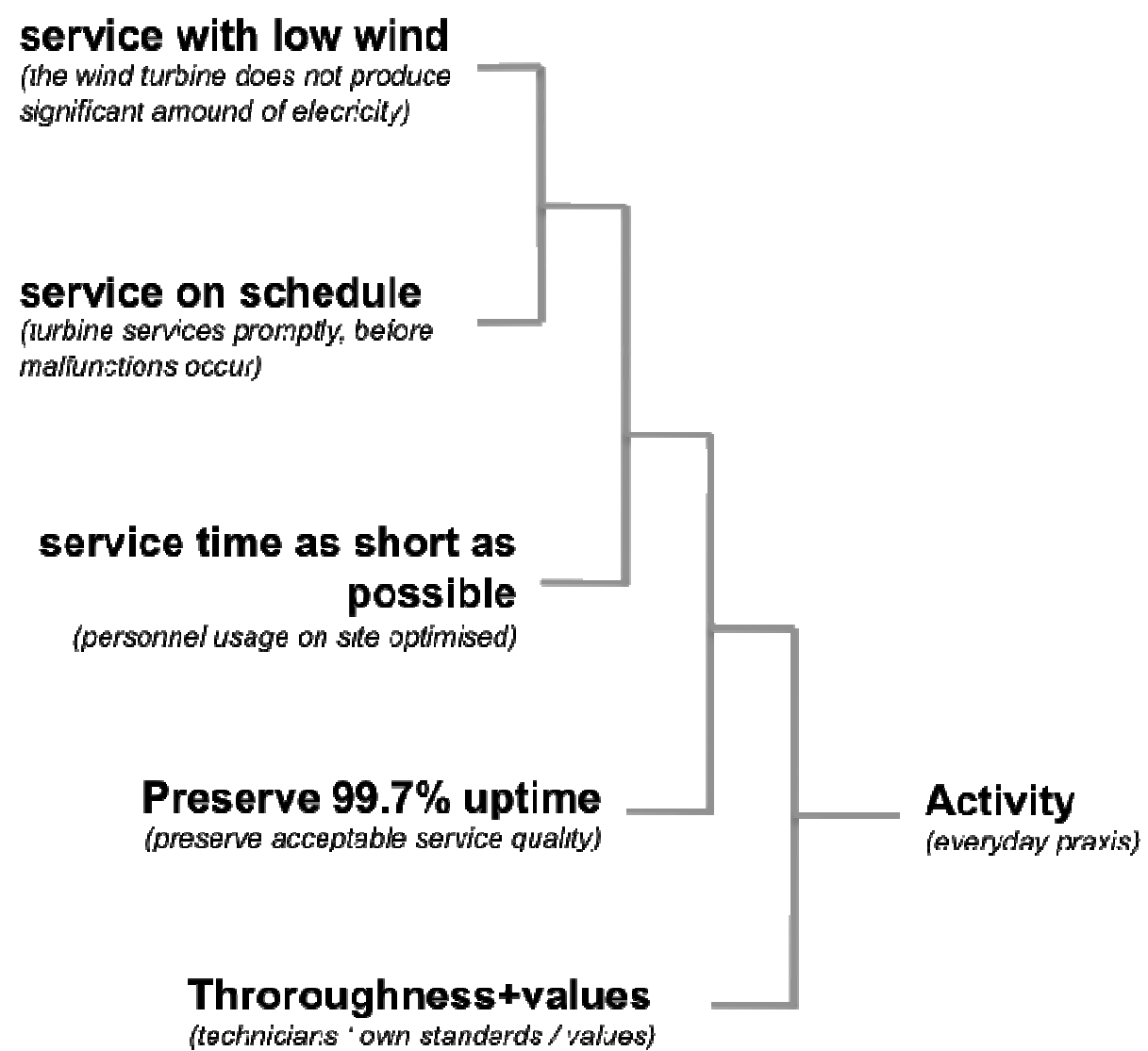

Fig.2. Demonstration of Contradiction Analysis for Wind Turbine Maintenance tasks

The resolution of this nested set of contradictions can be considered as the core of the service process, defining a frame to work on, when trying to intervene on that process.

\section{Discussion}

Developing such a contradiction analysis is neither straight forward as a process nor unequivocal in its results across different analysts. After all each ergonomics intervention is a construction and as such it always develops in an original and not replicable way.
Nevertheless we have been surprised how representations such as the above made immediate sense between different ergonomists but also and more importantly that they were accepted as relevant and meaningful by most stakeholders in the projects above.

We came to believe that if an ergonomist develops a perspective of activity regulation as coping within a space of diverging and often conflicting forces, an activity model can easily be depicted, which can be backed up theoretically, with many benefits for the practice of ergonomics: (a) better communication with the operator(s) under study, etc. (b) sets out the 
backbone for prioritizing issues and further analysis. (c) intervention with an increased level of relevance for the phenomenology of work as it affects directly what the operator(s) does, faces, controls, is afraid off.

In fact, when we propose to "chase after contradictions" as a strategy for swift results, we are referring to many different phenomena at the same time. For the time being we cannot provide concrete methodological guidance on the process. We can only point to the following prioritized hints: First and foremost the analyst should search for issues that can be identified -with insider's aid- as persistently significant for the worker(s) at a particular work situation (i.e. the main articulable concerns of the worker(s) at a particular moment and time frame). Second, the analyst should search for issues that can be identified as invariant bipoles of the objective (extrinsic) structure of the work situation (which not only constrain but have a persisting, determining effect on worker's conduct).

Third, he should pay attention to observed phenomena such as oscillations in worker's conduct, uneasiness', fluctuation in the work results etc. which may hide unarticulated or non-articulable concerns.

All the above can be attributed either to extrinsic factors of the work situation (formal goal structure, external demands vs internal needs etc.) or as understanding of what is -believed to be- experienced by the working individual or collectivity. The above hints are in fact just alternative ways of approaching contradictions i.e. techniques that can be employed for the initial steps of "contradiction analysis".

Since the method is still vague and immature, it is very easy for an analyst to commit the error and consider contradictions that go beyond his professional concern, i.e. contradictions involving or being mainly- resolvable at higher levels or to interpret any minor issue that he encounters as a contradiction. The art of this trade for the analyst is, on the one hand, to stay inside the envelope of his possibilities for a positive impact (e.g. to self restrain from elevating his contradiction analysis to a political or societal level) and, on the other hand, to restrain from getting drowned in the dialectics of self evident reality.

\section{References}

[1] De la Garza, C. \& Weill-Fassina, A. (2000). Régulations horizontales et verticales du risque. In A. Weill-Fassina \& T.H. Benchekroun (Eds), Approches ergonomiques du travail collectif dans les systèmes socio-techniques (p. 271-234). Toulouse : Octares

[2] Dejours, C. (1995). Le facteur humain. Paris : PUF

[3] Faverge, J.-M. (1966). L'analyse du travail en terme de régulation. In J.-M. Faverge, M. Olivier, J. Delahaut, P. Stephaneck \& J.C. Falmagne, L'ergonomie des processus industriels (p. 33-60). Bruxelles : Éditions de l'Institut de Sociologie

[4] Hollnagel, E. (204). Barrier analysis and accident prevention. Aldershot, UK: Ashgate

[5] Leplat, J. (2000). L'analyse psychologique de l'activité en ergonomie. Toulouse : Octarès

[6] Nardi, Bonnie A. (1996). Context and Consciousness: Activity Theory and Human-computer Interaction. pp 1-20. MIT Press, Cambridge, MA. ISBN 0-262-14058-66

[7] Rasmussen, J., Pejtersen, A.M., Goodstein, L.P., 1994. Cognitive Systems Engineering. John Wiley \& Sons, NewYork

[8] Sperandio, J.-C. (1972). Charge de travail et régulation des processus opératoires. Le Travail $\mathrm{Hu}-$ main,

$35,85-93$. 